\title{
VERA Installation Guide
}

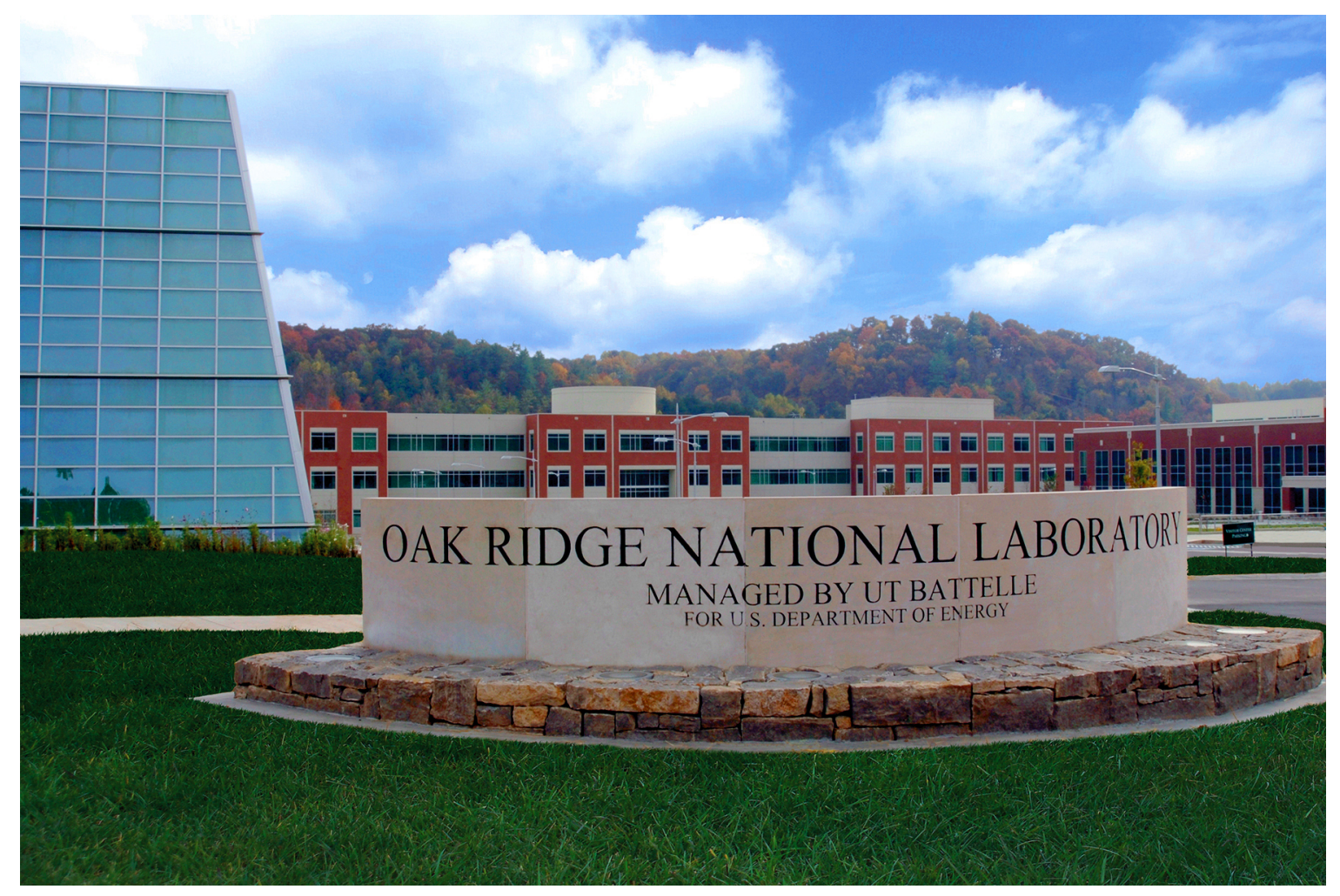

Mark Baird

Roscoe A. Bartlett

Ron Lee

Brenden T. Mervin

Benjamin S. Collins

Robert A. Lefebvre

April 2021

\section{OAK RIDGE \\ National Laboratory}




\section{DOCUMENT AVAILABILITY}

Reports produced after January 1, 1996, are generally available free via US Department of Energy (DOE) SciTech Connect.

Website: www.osti.gov/

Reports produced before January 1, 1996, may be purchased by members of the public from the following source:

National Technical Information Service

5285 Port Royal Road

Springfield, VA 22161

Telephone: 703-605-6000 (1-800-553-6847)

TDD: $703-487-4639$

Fax: 703-605-6900

E-mail: info@ntis.gov

Website: http://classic.ntis.gov/

Reports are available to DOE employees, DOE contractors, Energy Technology Data Exchange representatives, and International Nuclear Information System representatives from the following source:

Office of Scientific and Technical Information

PO Box 62

Oak Ridge, TN 37831

Telephone: 865-576-8401

Fax: 865-576-5728

E-mail: report@osti.gov

Website: http://www.osti.gov/contact.html

This report was prepared as an account of work sponsored by an agency of the United States Government. Neither the United States Government nor any agency thereof, nor any of their employees, makes any warranty, express or implied, or assumes any legal liability or responsibility for the accuracy, completeness, or usefulness of any information, apparatus, product, or process disclosed, or represents that its use would not infringe privately owned rights. Reference herein to any specific commercial product, process, or service by trade name, trademark, manufacturer, or otherwise, does not necessarily constitute or imply its endorsement, recommendation, or favoring by the United States Government or any agency thereof. The views and opinions of authors expressed herein do not necessarily state or reflect those of the United States Government or any agency thereof. 
Nuclear Energy and Fuel Cycle Division

\title{
VERA INSTALLATION GUIDE
}

\author{
Mark Baird \\ Roscoe A. Bartlett \\ Ron Lee \\ Brenden T. Mervin \\ Benjamin S. Collins \\ Robert A. Lefebvre
}

April 2021

\author{
Prepared by \\ OAK RIDGE NATIONAL LABORATORY \\ Oak Ridge, TN 37831-6283 \\ managed by \\ UT-Battelle LLC \\ for the \\ US DEPARTMENT OF ENERGY \\ under contract DE-AC05-00OR22725
}





\section{CONTENTS}

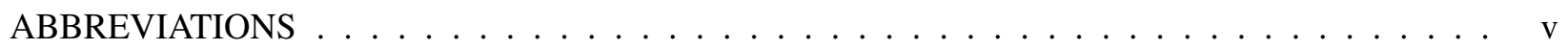

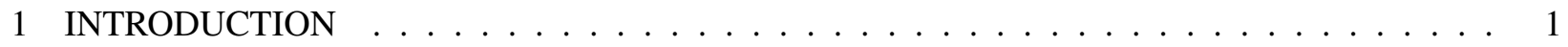

2 STANDARD VERA DEV ENV DIRECTORY STRUCTURE $\ldots \ldots \ldots \ldots \ldots \ldots \ldots$

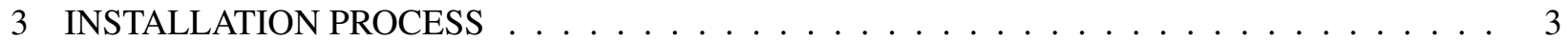

3.1 MAKE SURE THE BASIC PREREQUISITES ARE SATISFIED . . . . . . . . . . 3

3.2 DETERMINE SOURCE, SCRATCH, AND INSTALL DIRECTORIES $\ldots \ldots \ldots \ldots$

3.3 GET THE BASE VERA AND TRIBITS SOURCE DIRECTORIES $\ldots \ldots \ldots \ldots . \ldots$.

3.4 INSTALL THE BASE DEVELOPMENT ENVIRONMENT $\ldots \ldots \ldots \ldots \ldots \ldots$

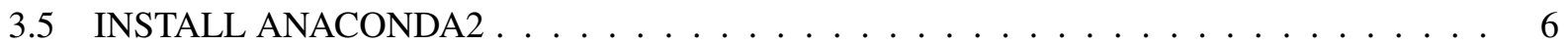

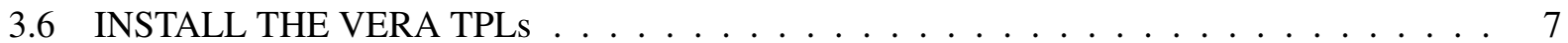

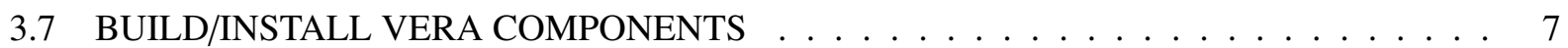

3.8 FINAL SETUP OF INSTALLED VERA DEV ENV AND FINAL CLEANUP . . . . . . . . 9

4 DETAILS ON INITIAL SETUP $\ldots \ldots \ldots \ldots \ldots$

4.1 REQUESTING ACCESS TO VERA REPOSITORIES $\ldots \ldots \ldots \ldots \ldots$

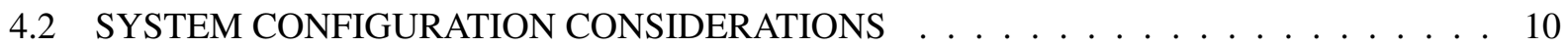

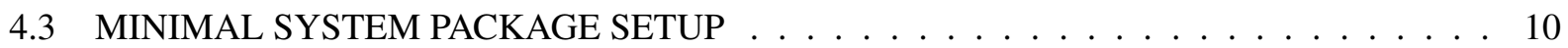

4.4 SSH SETUP FOR ACCESSING CODE-INT $\ldots \ldots \ldots \ldots \ldots$

4.5 CREATE UNIX USER AND GROUP $\ldots \ldots \ldots \ldots$

4.6 SET UP BASE DIRECTORIES FOR VERA $\ldots \ldots \ldots \ldots$

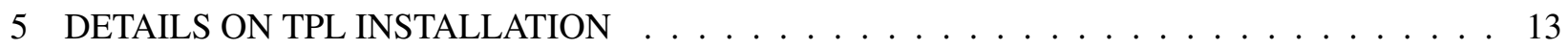

6 DETAILS VERA COMPONENT BUILD, TEST, AND INSTALLATION . . . . . . . . . . . . . 14

6.1 LOAD VERA DEV ENV . . . . . . . . . . . . . . . . . . . . . . . . . 14

6.2 CLONE REMAINING VERA COMPONENTS . . . . . . . . . . . . . . . . . . 14

6.3 CHECKING OUT A SPECIFIC VERSION OF VERA . . . . . . . . . . . . . . 15

7 DETAILS ON FINALIZING VERA DEV ENV INSTALLATION $\ldots \ldots \ldots \ldots . \ldots \ldots$

8 DETAILS ON INSTALLING VERA $\ldots \ldots \ldots \ldots \ldots \ldots \ldots \ldots$

8.1 GET SOURCE FOR VERA COMPONENTS TO INSTALL . . . . . . . . . . . . 18

8.2 CONFIGURE, BUILD, AND TEST VERA COMPONENTS TO INSTALL . . . . . . . . . 18

8.3 INSTALL BUILT VERA COMPONENTS . . . . . . . . . . . . . . . . . . . . . . . 19

8.4 DOCUMENTATION FOR INSTALLED VERA COMPONENTS . . . . . . . . . . . . . 19

ADDITIONAL INSTALLATION INFORMATION . . . . . . . . . . . . . . . A-1

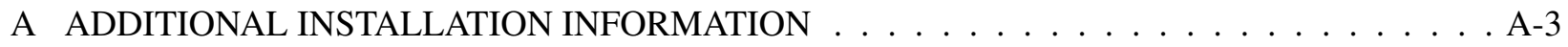

A.1 SET UP REMOTE SSH TUNNEL . . . . . . . . . . . . . . . . . . . . . . A-3

A.2 MINIMAL SYSTEM PACKAGE SETUP ON VARIOUS SYSTEMS $\ldots \ldots \ldots \ldots$. . A-4

A.3 OFFICIAL VERA TPL VERSIONS $\ldots \ldots \ldots \ldots \ldots \ldots \ldots \ldots$ A-5 $\ldots \ldots \ldots$

A.4 SHARED VS. STATIC LIBRARIES $\ldots \ldots \ldots \ldots \ldots \ldots$. . . . . . . . . . . . 



\section{ABBREVIATIONS}

CASL Consortium for Advanced Simulation of Light Water Reactors

GCC GNU Compiler Collection

HPC high-performance computing

ORNL Oak Ridge National Laboratory

SSH Secure Shell Protocol

TPLs third-party libraries

triBITS tribal Build, Integrate, and Test System

VUG VERA Users Group 


\section{INTRODUCTION}

This guide describes the structure and setup of the standard Virtual Environment for Reactor Applications(VERA) development environment (VERA Dev Env) and standard VERA third-party libraries (TPLs) that need to be in place before many of the VERA simulation components are installed. It describes everything from the initial setup on a new machine to the final build, testing, and installation of VERA components. The goal of this document is to describe how to create the directories and contents outlined in Section 2, obtain the remaining VERA source, and build, test, and install any of the necessary VERA components on a given system. This document describes the process for a development version of VERA and for a released tarball of the VERA sources.

Section 2 outlines the directory structure. Section 3 describes the the major steps of the installation process. Section 3 contains all of the information needed to perform the full install of the VERA Dev Env, as well as the install of VERA components themselves. The remaining sections contain more information and details for variations, as well as tips for how to solve problems when things go wrong.

WARNING: This guide describes installation of the VERA Dev Env and TPLs; it does not contain specific information about specific VERA simulation components. That information is found in other sources. Please consult with a VERA Users Group (VUG) representative about what VERA components are available to install from source and what capabilities the components provide. In this manual, information about other VERA repositories is only used for examples and may not be up to date.

\section{STANDARD VERA DEV ENV DIRECTORY STRUCTURE}

The standard directory structure for the installation of the VERA Dev Env is given below:

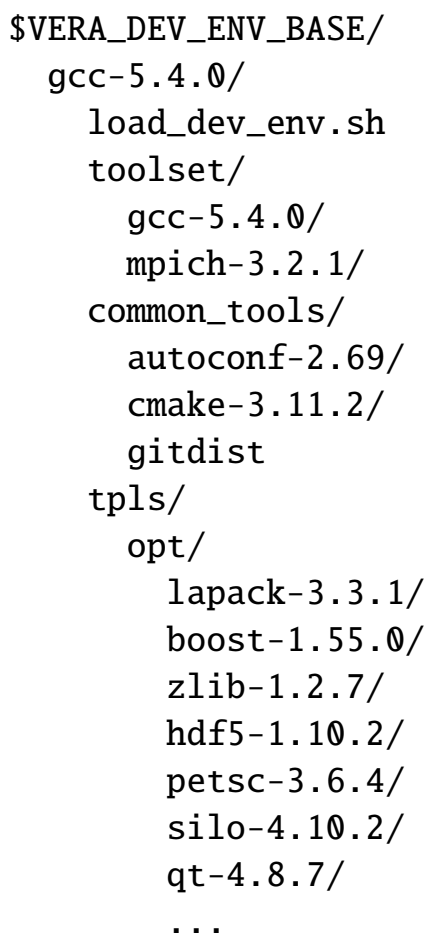




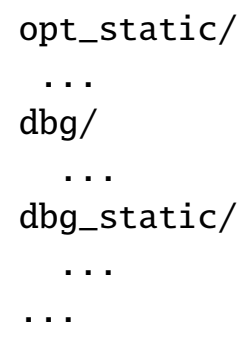

For the example in this guide,

VERA_DEV_ENV_BASE=/projects/vera

was set, but note that any base directory can be used. This directory is where the prerequisite TPLs (see A.1) and VERA tools that are used for configuring, building, testing, and installing VERA are deployed.

For the standard GNU Compiler Collection (GCC) 5.4.0 VERA Dev Env,

VERA_DEV_ENV_COMPILER_BASE=\$VERA_DEV_ENV_BASE/gCc-5.4.0

was set.

For a given compiler set, TPLs can be installed for different configurations, such as debug (dbg), optimized (opt), or other variations (e.g., dbg-checkedstl). The standard TPL install is used in this guide and is provided below:

VERA_TPL_INSTALL_DIR=\$VERA_DEV_ENV_COMPILER_BASE/tpls/opt

For the install of static TPLs, use:

VERA_TPL_INSTALL_DIR=\$VERA_DEV_ENV_COMPILER_BASE/tpls/opt_static

Shared libraries should be the default (see A.4).

All of the VERA Dev Env, TPL install tools, and other directory environments use the variables and typical values shown in Table 1 to determine a particular installation of the VERA Dev Env to use for building/testing/installing VERA components.

Table 1. VERA directory environment variables

\begin{tabular}{|l|l|}
\hline Variable & Common/Example Value \\
\hline VERA_DEV_ENV_BASE & /projecrts/vera \\
\hline VERA_DEV_ENV_COMPILER_BASE & \$VERA_DEV_ENV_BASE/gcc-5.4.0 \\
\hline VERA_TPL_INSTALL_DIR & \$VERA_DEV_ENV_COMPILER_BASE/tpls/opt \\
\hline VERA_BASE_DIR & \$HOME/VERA.base \\
\hline VERA_SCRATCH_DIR & \$VERA_BASE_DIR/scratch \\
\hline VERA_DIR & \$VERA_DEV_ENV_BASE/vera-X.Y.Z-Source \\
\hline VERA_BUILD_DIR & \$HOME/VERA_BUILD \\
\hline VERA_INSTALL_DIR & /projects/vera/vera_installs/ date +\%Y-\%m-\%d \\
\hline
\end{tabular}

WARNING: When using shared libraries, be careful to avoid the error "RegularExpression::compile(): Expression too big." when using RPATH and when RPATH gets stripped out on install. To avoid this error, use the shortest build directory possible, such as the following: 
export VERA_BUILD_DIR=\$HOME/VERA_BUILD

\section{INSTALLATION PROCESS}

This section gives the set of commands that must be run to install the VERA Dev Env and perform a test build of VERA. These instructions assume that the entire VERA Dev Env will be installed, starting with GCC, MPI, CMake, and TPLs. Customization can be done as needed, but it is not covered here. All the sources and scripts are provided as downloads from the internet or from networked computers, or they are provided as compressed files (e.g., on compact discs for those who cannot access outside machines from the install machines). Links for additional details are given for each step below, or they are available in the install tools themselves.

The steps are as follows:

1. Make sure the basic prerequisites are satisfied (Section 3.1).

2. Determine source, scratch, and install directories (Section 3.2).

3. Get the base VERA and Tribal Build, Integrate, and Test System (TriBITS) source directories (Section 3.3).

4. Install the base development environment (Section 3.4)

5. Install Anaconda2 (Section 3.5).

6. Install the VERA TPLs (Section 3.6).

7. Build/install VERA Components (Section 3.7).

8. Complete setup of the installed VERA Dev Env, and perform final cleanup (Section 3.8).

\subsection{MAKE SURE THE BASIC PREREQUISITES ARE SATISFIED}

Before the VERA development/install environment and VERA itself can be installed, the following steps should be performed:

1. Create a vera-admin Unix user, a vera-users Unix group, and the required base directories for building, testing, installing, and maintaining VERA (see Sections 4.5 and 4.6).

2. Make sure the system can handle an installation of VERA (see Sections 4.2 and 4.3).

3. If the source is from the VERA Git repositories:

a. Set up Secure Shell Protocol (SSH) access to the Oak Ridge National Laboratory (ORNL) GitLab website code-int . ornl . gov (casl-dev for short, see Section 4.4 and A.1).

b. Get approval to access the required protected VERA git repositories (see Sections 4.1 and 6.2). 


\subsection{DETERMINE SOURCE, SCRATCH, AND INSTALL DIRECTORIES}

Set environment variables for the location of the installed/shared VERA development/install environment VERA_DEV_ENV_BASE and the base location of the VERA sources VERA_BASE_DIR to drive the INSTALL and other specified in Table 1. For example:

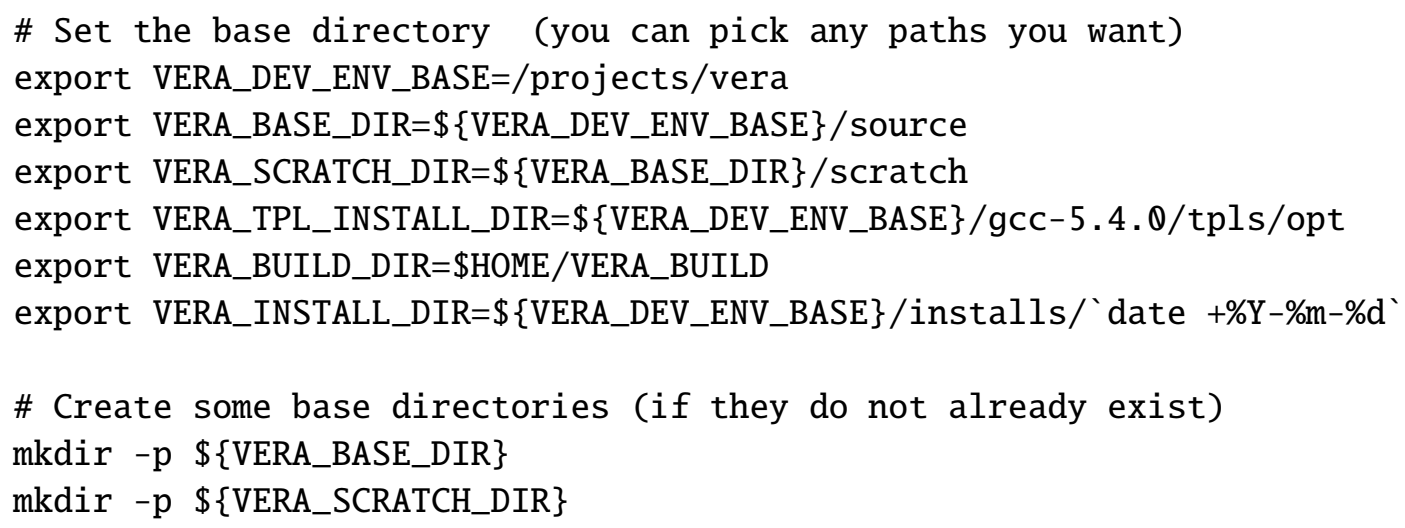

All the other directories will be created as described below, or they will be created automatically by running various install tools.

WARNING: When using shared libraries, be careful to avoid the error "RegularExpression::compile(): Expression too big." when using RPATH and when RPATH gets stripped out on install. To avoid this error, use the shortest possible build directory, such as:

export VERA_BUILD_DIR=\$HOME/VERA_BUILD

\subsection{GET THE BASE VERA AND TRIBITS SOURCE DIRECTORIES}

If using a VERA tarball, enter:

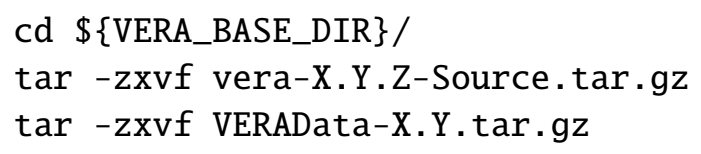

then set:

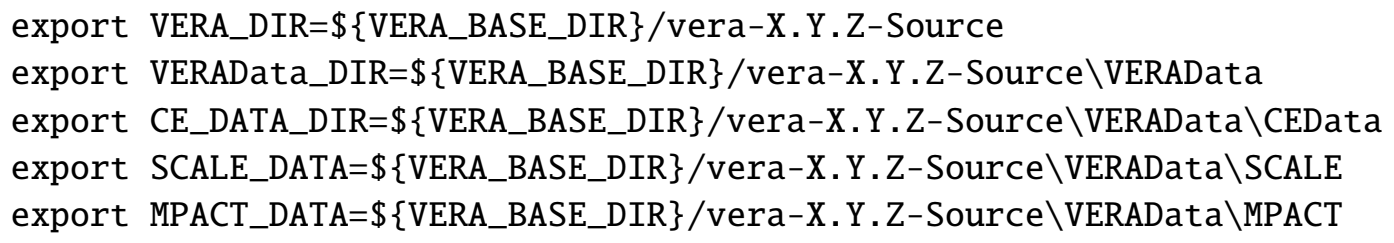

If cloning the sources from code-int: VERA (if they are not already cloned), first set up the SSH tunnel (see A.1) to code-int with:

$$
\text { ssh -fN tunnelinit }
$$

Then clone with the following commands: 
cd \$ \{VERA_BASE_DIR $\} /$

git clone git@code-int:VERA/VERA

cd VERA/

git clone git@code-int:VERA/TriBITS

Then set:

export VERA_DIR $=\$\{$ VERA_BASE_DIR $\} /$ VERA

\subsection{INSTALL THE BASE DEVELOPMENT ENVIRONMENT}

To install all the generic tools (excluding the TPLs), run the following commands:

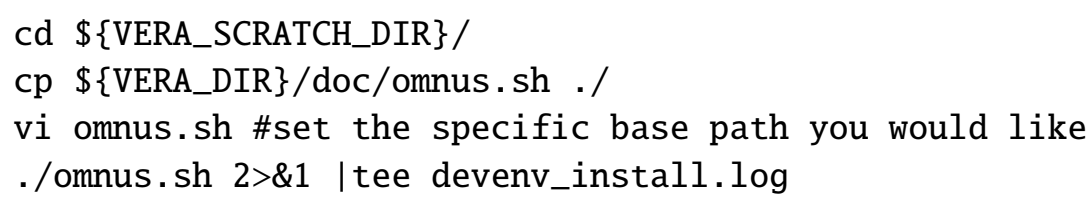

If internet access to download source libraries is unavailable, then use the omnus_local. sh script and the env source files (vera-4.2_env.tar.gz), which can be provided by request. To request the vera-4.2_env.tar.gz file, email casl-vri-infrastructure@casl.gov. After the file is received, run the following command(s):

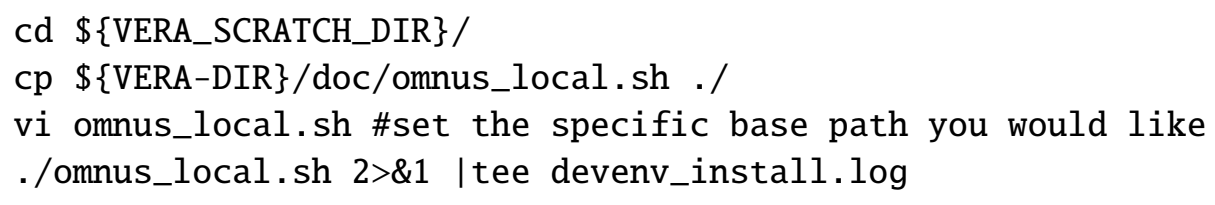

- If any error occurs, check the log file (devenv_install.log) to see what failed, and then check the $\log$ file it indicates to see the actual errors.

- If the tool chain is being built with GCC 5.X and an error is encountered when compiling the VERA version of GCC that contains

$$
\text { ...version 'GLIBCXX_3.4.20' not found..., }
$$

then the $\mathrm{C}++$ standard library must be preloaded using the following command:

export LD_PRELOAD=/usr/lib/libstdc++.so. 6

Note that the path to the library may be different for your system, depending on the base install path systems. Also note that this environment variable must be added to the development and runtime environments for VERA (i.e., the \$ $\{$ VERA_DEV_ENV_COMPILER_BASE $\} /$ load_dev_env.sh and the \$\{VERA_INSTALL_DIR\}/load_env.sh scripts).

Once the omnus script completes, it should install GCC 5.4.0, MPICH 3.2.1, CMake 3.14.3, gitdist, and load_dev_env.sh, as shown in Section 2.

After a successful install, source the installed load_dev_env.sh script as: 
source \$\{VERA_DEV_ENV_BASE\}/gcc-5.4.0/1oad_dev_env.sh

Then, pre-pend PATH to find the installed programs (cmake, gitdist, gcc, mpicc, ...) in the locations shown in Section 2. Make sure to source the new development environment, with, for example

which cmake

which gcc

which mpicc

This should return

$\ldots /$ gcc-5.4.0/common_tools/cmake-3.14.3/bin/cmake

$\ldots /$ gcc-5.4.0/toolset/gcc $-5.4 .0 / \mathrm{bin} / \mathrm{gcc}$

.../gcc-5.4.0/toolset/mpich-3.2.1/bin/mpicc

\subsection{INSTALL ANACONDA2}

The installation of Anaconda2 is required. It provides an updated Python version, as well as numpy and other Python modules used by several VERA packages. Also, if the user plans to utilize the VERArun tool on high-performance computing (HPC) systems, then the additional packages are required for a successful setup.

To install Anaconda2, go to the site

- https://docs.anaconda.com/anaconda/install/

and follow the instructions to download the binary installation script for the version based on Python 2.7.13. For 64-bit Linux systems, use the following script:

- https://repo.anaconda.com/archive/Anaconda2-5.3.1-Linux-x86_64.sh

The URL location may change.

Once this binary install script is downloaded (presumably to /Download), then run it as

chmod $\mathrm{u}+\mathrm{x} \sim /$ Downloads/Anaconda2-5.3.1-Linux-x86_64.sh

/Downloads/Anaconda2-5.3.1-Linux-x86_64.sh

When the interactive install script asks for an install location, enter the following:

\$VERA_DEV_ENV_BASE/gcc-5.4.0/common_tools/anaconda2 Note that \$VERA_DEV_ENV_BASE/

must be expanded manually. Users can choose to allow the script to edit their $\sim /$. bashrc file or not to add this to their PATH. However, later in this document, it is assumed that that step is not done. Users'

load_dev_env file should address this.

To use the installed Anaconda2 Python executable, related modules, and tools, update your dev env path to the following:

export PATH=\$VERA_DEV_ENV_BASE/gcc-5.4.0/common_tools/anaconda2/bin: \$PATH

Verify that the correct version of Python is being used by running

which python

python --version 
which returns

.../gcc-5.4.0/common_tools/anaconda2/bin/python

Python 2.7.13 : : Anaconda 4.3.1 (64-bit)

Before doing any configures, builds, or tests with VERA, this path must be prepended (as described earlier), or the load_dev_env file will need to be sourced.

\subsection{INSTALL THE VERA TPLS}

First, obtain the vera_tpls source repository and the matching version of the TPLs:

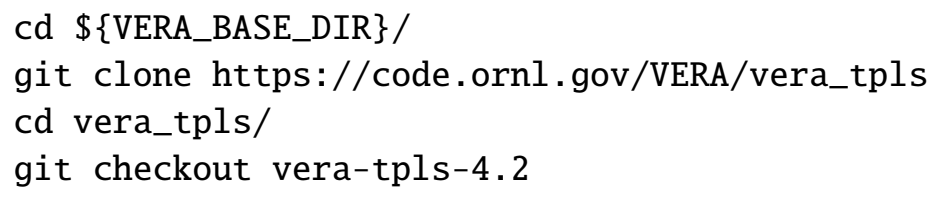

If users do not have access to GitHub, they can obtain the tarball vera_tpls-4 .2 . tar.gz and untar/unzip it in the current directory using

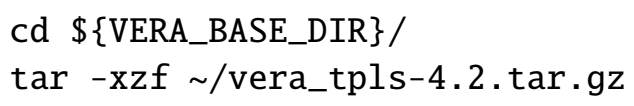

Once the directory vera_tpls for the correct version of the VERA TPLs is obtained (using one of the methods mentioned earlier), the TPLs can be configured and built with

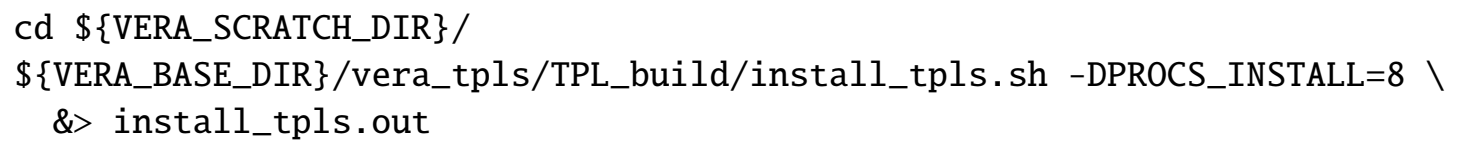

NOTE: Adjust -DPROCS_INSTALL=8 to the appropriate number of processes for the current machine, but please be respectful of fellow system users.

By default, this installs a shared library version of the TPLs (see Section A.4). A static library version can be installed by entering -DENABLE_SHARED=OFF and passing in

-DCMAKE_INSTALL_PREFIX=\$VERA_DEV_ENV_COMPILER_BASE/tpls/opt_static

For more details, see Section 5.

\subsection{BUILD/INSTALL VERA COMPONENTS}

Finally, the installed VERA Dev Env must be tested by configuring, building, testing, and installing VERA from source (installation not required).

If the VERA source were obtained from a source tarball file (see earlier in this document), then all of the sources needed to build the VERA components should be in place (pointed to by \$ VVERA_DIR\}).

If the VERA source were obtained from the Git repositories on code-int, then the remaining VERA Git repositories must be cloned. First, if an SSH tunnel is necessary, initialize the tunnel (see Section A.1) with 


$$
\mathrm{ssh}-\mathrm{fN} \text { tunnelinit }
$$

Then the remaining repositories can be cloned using:

cd \$\{VERA_DIR $\} /$

./clone_vera_repos.py

Once the source is obtained, users can configure, build, test, and install with

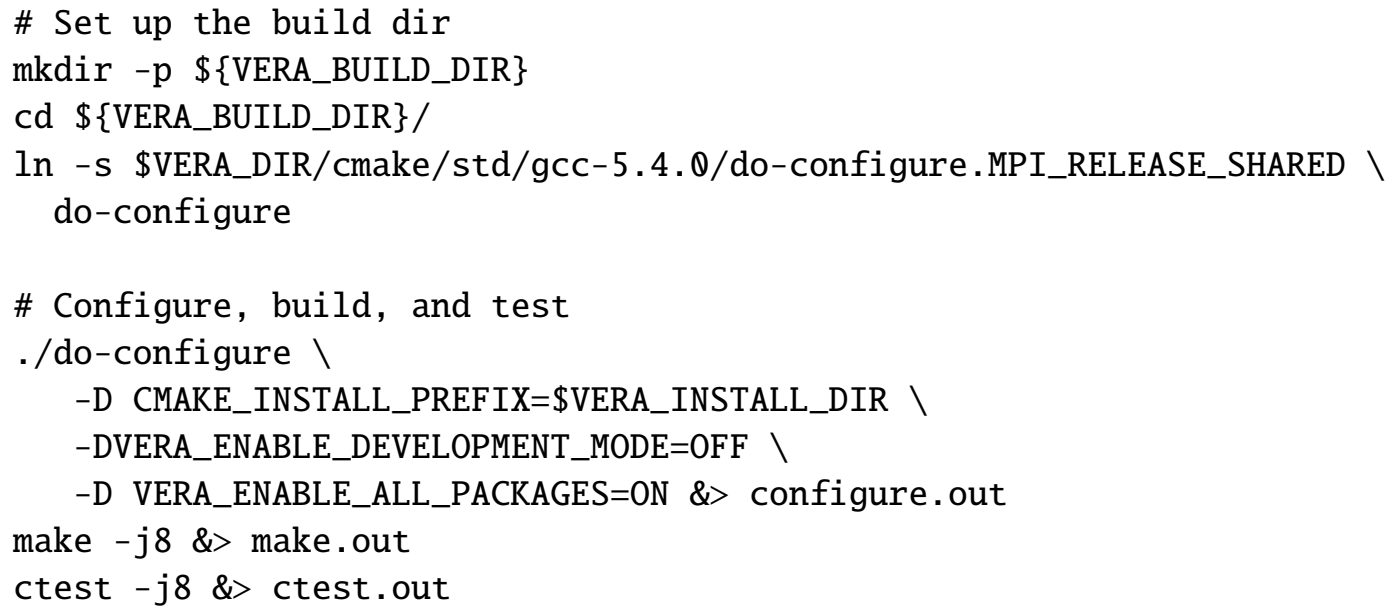

NOTE: The above input shows the usage of 8 processes to build the code and run the tests. Change 8 to the appropriate value for the machine being used.

If all of the tests pass and the output from ctest looks like the following

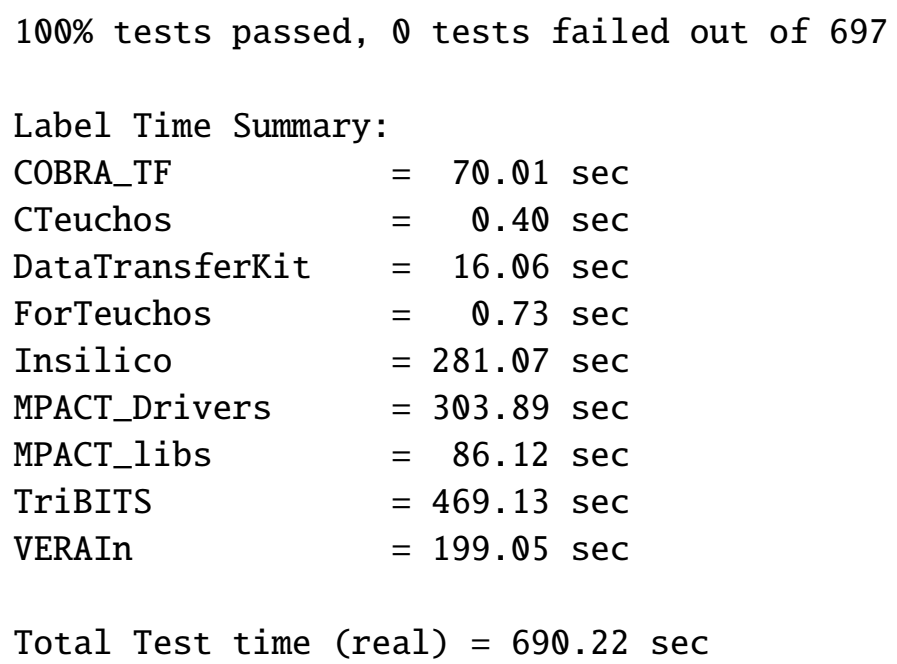

then all is well. The number of tests may vary based on enables and test updates.

VERA can be installed for use by others with

make $-j 8$ install \&> make.install.out

Permissions on the installed version of VERA should be set using 
chmod chgrp -R vera-users \$VERA_INSTALL_DIR

(see Section 4.5.)

After the install, see the instructions using VERA in the readme file:

\$VERA_INSTALL_DIR/README

In particular, users should source the following script to set up their environment (i.e., PATH and other variables) to run the installed executables and scripts:

\$VERA_INSTALL_DIR/load_env.sh

See the online copy README.VERA (becomes the installed file README).

NOTE: The commands shown above are used to build and install a shared library version of VERA (see Section A.4). A static library version can be installed by replacing MPI_RELEASE_SHARED with MPI_RELEASE_STATIC above. Users may want to use this approach if they are installing static TPLs using -DENABLE_SHARED=OFF in Section 3.6.

\subsection{FINAL SETUP OF INSTALLED VERA DEV ENV AND FINAL CLEANUP}

After the basic tools and TPLs of the VERA Dev Env (basic tools and TPLs) have been installed, the directories must be opened to provide other users with access to the installed tools and libraries. Since there is no export-controlled information or other sensitive data contained in the installed VERA Dev Env, it can be made public by entering

chmod -R a+rX \$ \{VERA_DEV_ENV_BASE $\}$

It is important to note that the SOURCE or BUILD directories should not be a sub directory of \$ \{VERA_SCRATCH_DIR\}, or else unlicensed users may have access. Extreme caution should be followed with the SOURCE and BUILD directory access.

Once users have finished installing and testing the VERA Dev Env, the generated texttt*.out and *. $\log$ files should be saved to archive details about the VERA Dev Env install for later reference. At this point, the scratch directory $\$\{$ VERA_SCRATCH_DIR $\}$ can be deleted.

More details about VERA and the installation of the VERA Dev Env are given in the following sections.

\section{DETAILS ON INITIAL SETUP}

Before any VERA-specific prerequisites can be installed, some initial setup is required. This section describes some of the tasks that must be performed to set up a machine so that it can be used to install the VERA Dev Env, clone the VERA repositories (or just unzip the VERA sources from a tarball), and build the various VERA components from source.

\subsection{REQUESTING ACCESS TO VERA REPOSITORIES}

If VERA is being installed from a release tarball, then users already have the source files. However, access must be granted if VERA is being pulled from the Git repositories on code-int.ornl.gov. Access to the 
repositories also requires that an ORNL Universal Computer Access Access Management System (UCAMS) account be added to the GitLab site code-int, and explicit access is also required to the Git repositories in accordance with the VERA Technology Control Plan. Users must request access from their VUG representative and provide a list of the specific VERA Git repositories (or VERA components) needed.

\subsection{SYSTEM CONFIGURATION CONSIDERATIONS}

Before work begins, an accounting of the system resources should be made. For example:

- Make sure there are enough processors available for parallel compilation. It is assumed that there are at least eight cores available on which to run parallel builds, tests, or other jobs. If eight cores are not available, then users can reduce the parallel level, as will be obvious with each command.

- Use the fastest file storage system available for holding source code and for compiling and running test cases: try to avoid Network File System-mounted directories for compilation and testing.

Other considerations include:

- If the user is not building from a tarball, can the machine create SSH tunnels to download VERA and TPL repositories?

- Can the machine access the internet (e.g., gi thub.com)? If not, ensure that all packages needed are available in the VERA and/or TPL repositories from code-int or gzipped tar files using an SSH tunnel/ftp download.

- Can a remote user access the machine if troubleshooting assistance is needed?

\subsection{MINIMAL SYSTEM PACKAGE SETUP}

Before proceeding to install the various VERA prerequisites, the following software packages must be installed on the system using the system's native package manager:

- GCC gec C compiler (recent version is not necessary): builds the official version of GCC from source. The newer GCC version is used to build everything else.

- GCC $\mathbf{g + +} \mathbf{C + +}$ compiler (recent version is not necessary): builds CMake from source. The newer GCC $\mathrm{g}++$ version is used to build everything else.

- patch: supports CMake.

- texinfo: The makeinfo program is needed to install GCC from source.

- GNU M4: needed for the build of autoconf.

- Git (version 1.6.0 or newer): needed to clone several Git repositories.

- git-lfs (version 2.0 or newer): needed to clone several Git repositories.

- Python (version 2.7.9 or newer but not version 3.x): needed for the basic install scripts and some helper scripts used in VERA. 
- NumPy (version 1.8.0 or newer): used in the COBRA-TF test harness.

- bash: needed for some of the shell scripts that refer explicitly to bash.

- Perl (VERA is tested with version v5.10.1, but newer versions should also work): needed to build libmesh/MOOSE/Peregrine and to run the VERA input parser react2xml .pl.

- X11 (includes development libraries, as well as runtime libraries): needed to build and install the required VERA TPL QT.

- ZLIB (includes development libraries, as well as runtime libraries): needed to build and install the required VERA TPL HDF5.

- libcurl* (may not be installed by default on some systems): needed for the NetCDF TPL build, which is used by MOOSE/Bison.

The exact packages that users must install on different types of systems are specified in Section A.1.

Users will require many other software packages, but they should already be installed on any proper Linux/Unix system.

\subsection{SSH SETUP FOR ACCESSING CODE-INT}

To access the VERA repositories on code-int.ornl.gov, users must establish public/private SSH keys and register the public SSH with the code-int.ornl.gov GitLab website that is used to manage the VERA git repositories. In this guide, <ucams-id> is used to signify the user's 3-char ORNL UCAMS ID.

If the internal ORNL website code-int (code-int. ornl.gov) is not directly reachable from a user's machine (referred to as <your-machine $>$ in this document), a remote SSH tunnel to code-int must be established as described in Section A.1).

First, if no public/private SSH keys have been set up on the machine, then they must be set up with

$$
\$ \mathrm{~cd} \sim / . \mathrm{ssh} \& \& / \text { usr/bin/ssh-keygen -t rsa -b } 1024
$$

Several prompts will appear. The defaults should be accepted by pressing the $<$ ENTER $>$ key three times.

The public key that was just created, /.ssh/id_rsa.pub, must then be uploaded to GitLab using gocitrix.ornl.gov. To log in, open a browser (Chrome recommended), log in to gocitrix, open a browser within the session, and navigate to code-int.ornl.gov. Complete the registration, and under settings $>$ SSH keys, copy your RSA public key to the text box and save. Then, send an email to vera-support@ornl.gov and ask for permission to access the code-int repositories.

If a user is not on the ORNL network with direct access to the machine code-int, then the SSH tunnel must be opened code-int using

$$
\mathrm{ssh}-\mathrm{fN} \text { tunnelinit }
$$

(See Section A.1.) 


\subsection{CREATE UNIX USER AND GROUP}

To properly administer and protect VERA installations, it is recommended to set up the following:

- A vera-admin user: a Unix user account specifically for maintaining VERA installations.

- A vera-users group: a list of Unix users with access permission and a need to know for running the installed VERA components.

NOTE: The list of users added to vera-users must be given explicit permission to access all of the installed VERA components. If users are not sure who can be on this list, please contact vera-support@ornl.gov or another responsible representative from Consortium For Advanced Simulation Of Light Water Reactors (CASL) for guidance.

\subsection{SET UP BASE DIRECTORIES FOR VERA}

Once the vera-admin user account has been created, the \$ $\{$ VERA_DEV_ENV_BASE $\}$ and \$ $\{$ VERA_INSTALL_DIR\} directories must be created by the root user or a user with sudo privileges. Then, ownership of these directories must be transferred to the vera-admin user. This transfer can be performed using the following commands:

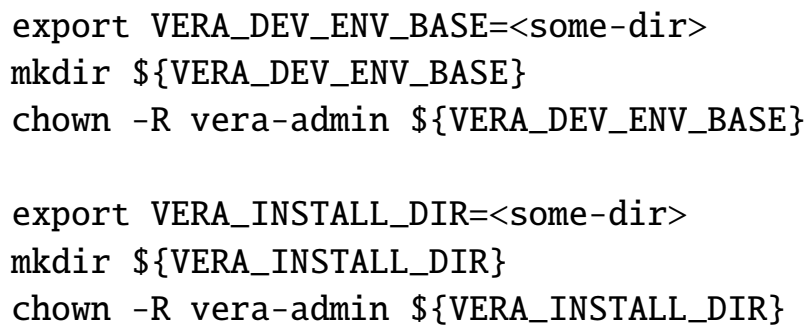

To reduce the need for root or sudo access during installation, a \$ $\{$ VERA_R00T_DIR\} containing the following directories can be used:

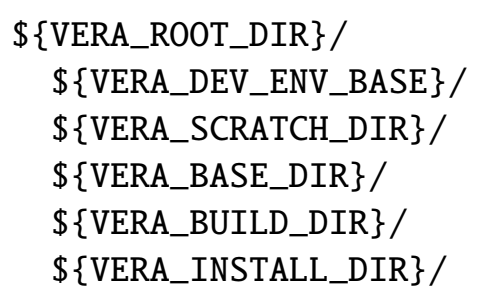

By using this directory structure, someone with only root or sudo access must perform the following operations:

1. Ensure that all of the required system packages have been installed.

2. Create the vera-admin user.

3. Create the vera-users group.

4. Create the \$ $\{$ VERA_ROOT_DIR\}.

5. Transfer ownership of $\$\{$ VERA_ROOT_DIR $\}$ to vera-admin. 
Once these actions have been performed, the vera-admin user will have all of the necessary permissions to build, test, install, and maintain the VERA installation.

\section{DETAILS ON TPL INSTALLATION}

A standard installation of VERA TPLs can be performed using the following command:

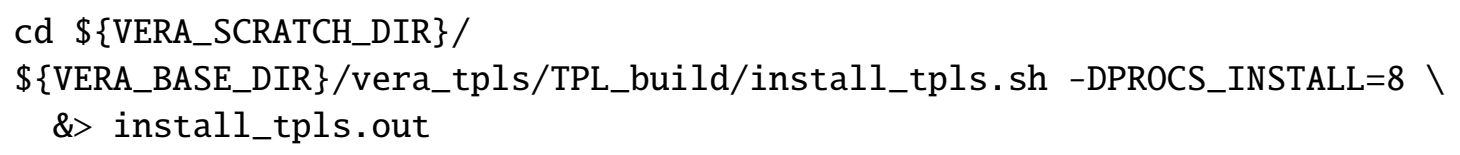

as described in Section 3.6. However, this is just a thin shell script that uses a CMake ExternalProject build of the TPLs.

The TPL build system can be pulled by using

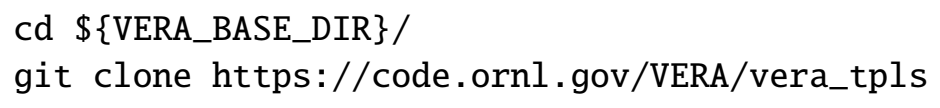

when pulling from GitHub.

The shell script install_tpls. sh creates a CMake binary directory:

\$\{VERA_SCRATCH_DIR $\} / T P L \_b u i l d /$

It then runs CMake, pointing to the CMake ExteranlProject directory:

\$\{VERA_BASE_DIR\}/vera_tpls/TPL_build/

This TPL_build CMake project accepts a number of CMake cache variables which include:

- CMAKE_BUILD_TYPE: Release or Debug

- ENABLE_SHARED: ON means that only shared *. so libraries will be installed, whereas OFF means that only static *. a libraries will be installed.

- CMAKE_INSTALL_PREFIX: base path to TPL install location; TPLs are installed under this directory, with one subdirectory for each TPL.

- TPL_LIST: list of TPLs to install; can be a comma-separated list. The full list of TPLs (and the default value for this variable) is

ZLIB ; HDF 5 ; NETCDF ; LAPACK ; QT ; PETSC ; SLEPC ; SILO ; SUNDIALS ; BOOST

Any subset of the TPLs can be listed and will be installed instead.

- PROCS_INSTALL: number of processors to use to build each TPL: at minimum of four is recommended because of the lengthy BOOST and QT build times. This is the last line path to vera_tpls/TPL_build repository subdirectory.

The install tool

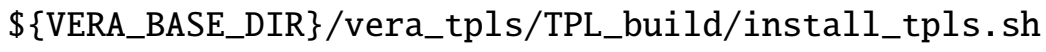


is a bash script that sets all of the defaults needed to perform the basic build, including compiler and other options. However, as shown in Section 3.6, users can pass in any CMake cache variable, and it will override the defaults set in install_tpls.sh. For example, to install static libraries in addition to shared libraries, and to use 16 processes, use

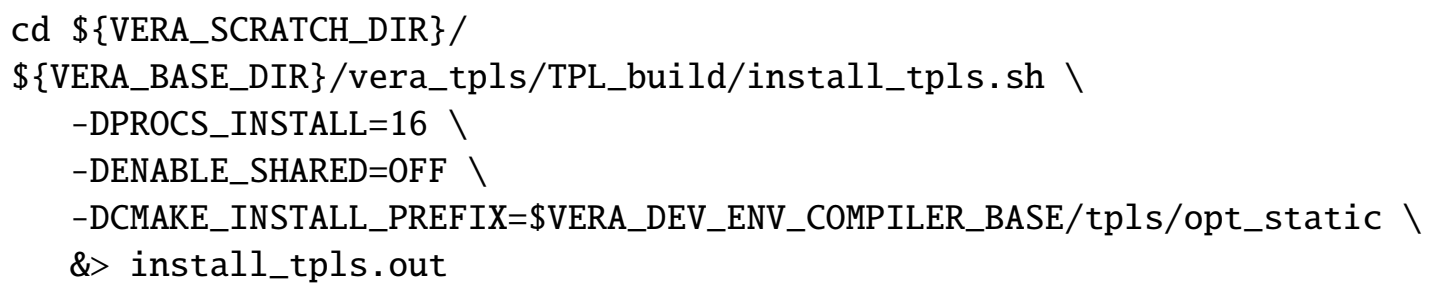

After the install script finishes calling cmake to configure the TPL build, it calls

make

to drive the build and install process.

Users can examine the script install_tpls. sh to see what it does, make needed modifications to the install process, and run the commands manually if needed.

\section{DETAILS VERA COMPONENT BUILD, TEST, AND INSTALLATION}

Once the VERA prerequisites (i.e., compilers, TPLs, other tools) have been installed, users must install the remaining VERA Git repositories for the desired components (if working from the version-controlled source), set up a build configuration, build, test, and finally, install. If users are working from an unzipped (release) tarball, then no extra git clones are needed, since all required source files will already be in place.

\subsection{LOAD VERA DEV ENV}

Before users can configure, build, test, and install any VERA component software, the installed VERA Dev Env must first be loaded into the user's shell. If it is not already loaded, then run

source \$\{VERA_DEV_ENV_COMPILER_BASE\}/load_dev_env.sh

export PATH=\$VERA_DEV_ENV_BASE/common_tools/anaconda2/bin: \$PATH

\subsection{CLONE REMAINING VERA COMPONENTS}

When working from a tarball distribution of VERA, there is nothing left to clone, so users can skip this section.

However, when working from the version-controlled sources, the remaining VERA Git repositories can be cloned, for example, with

cd \$ VVERA_DIR $\} /$

./clone_vera_repos.py 
NOTE: The exact list of repositories to be cloned greatly depends on which VERA components with what functionality are desired or needed from VERA. Such information is not provided in this document. Users should ask their CASL VERA contact about what repositories they need to clone for specific purposes.

NOTE: Users must be part of the GitLab group git@code-int, which protects a repository; otherwise, when users clone, they will get an error message, like:

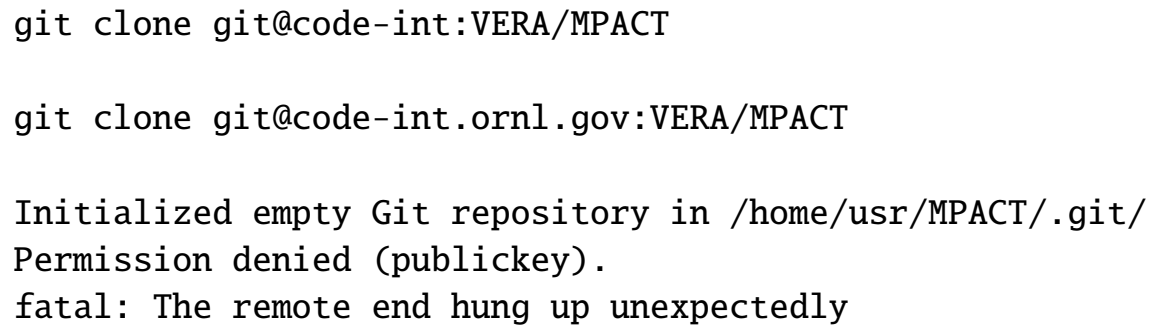

\subsection{CHECKING OUT A SPECIFIC VERSION OF VERA}

Users working from a tarball distribution of VERA can skip this section since the version is fixed.

However, when working from the Git repositories, various versions of VERA can be accessed.

The most recent version of VERA can be pulled using

$$
\begin{aligned}
& \text { cd } \$\{\text { VERA_DIR\}/ } \\
& \text { gitdist pull }
\end{aligned}
$$

This will pull the most recent version of VERA at that moment from the official development code-int: VERA $/<$ REPO $>/$ master branches. Although a rigorous, almost continuous integration process ensures that all basic automated tests pass before anything is pushed into the code-int/master branches, mistakes do occur, and there may be some more detailed acceptance tests that may not run successfully on any particular version of VERA at any moment for what is in code-int/master.

Therefore, to reduce the probability of pulling and using a defective version of VERA components, it is recommended that more specific versions of VERA be pulled since they have undergone more testing. More expensive automated acceptance tests run nightly and weekly, as well as some larger, manually run tests in some cases. A specific version of the VERA repositories can be checked out using the gitdist tool and a VERARepoVersion. txt file. A VERARepoVersion. txt file is created when VERA is configured from local Git repositories. The file is written to the VERA build tree, and it is installed in the base install tree. Every automated VERA build/test posted to the VERA CDash server includes exact Git version information in the generated VERARepoVersion. txt file. For a subset of VERA repos, a VERARepoVersion. txt file looks like

Before a user updates to a specific version of VERA, a VUG representative may provide the user with a specific VERARepoVersion.txt file which is typically named VERARepoVersion. <newdate>.txt (for some specific date $<$ newdate $>=$ YYYY-MM-DD). This file can be used to check out the specific version with

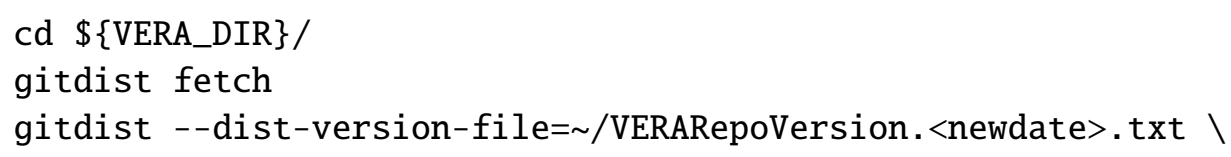


checkout _VERSION_

(see gitdist --help for more details).

This creates a "detached head"state for the local VERA Git repositories, and each repository will be at the exact commit listed in the VERARepoFileVersion. <newdate $>$. txt file. Below is a message that users might receive from each of the repositories:

Note: checking out '00149f1'.

You are in 'detached HEAD' state. You can look around, make experimental changes and commit them, and you can discard any commits you make in this state without impacting any branches by performing another checkout.

If you want to create a new branch to retain commits you create, you may do so (now or later) by using - $b$ with the checkout command again. Example:

git checkout -b new_branch_name

HEAD is now at $00149 f 1 \ldots$ Merge remote branch 'origin/master' into rsicc_2013_tarball_refactor_3104

This is not a troublesome state for the purposes of building the source.

Also, by using the VERARepoVersion. txt file for a previous install, users can see what repositories have been changed and what commits have been added. For example, to compare to an older install in

\$\{VERA_DEV_ENV_BASE $\} /$ vera/<olddate $>$ /

users can compare with the new recommended version by running

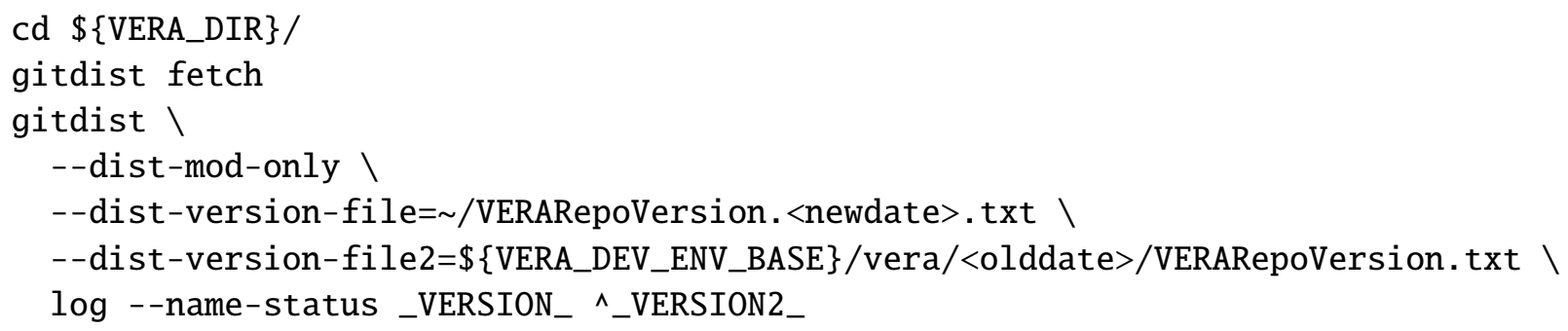

Many other types of Git commands can be used to supply one or two of the repository versions through a VERARepoVersion.txt file.

\section{DETAILS ON FINALIZING VERA DEV ENV INSTALLATION}

Once users have finished installing the VERA prerequisites consisting of the compilers, MPICH, other tools, and a complete set of TPLs shown in Section 2, and once users are finished testing the installs by building and testing needed VERA components, they must to set the permissions on the installed files and directories. 
The directory permissions for the Unix tools, compilers, and TPLs can be opened up for all to use. This can be accomplished with the following command:

$$
\text { chmod }-\mathrm{R} \text { a+rX <some-dir }>
$$

For example, world-readable permissions can be assigned using the following commands:

$$
\text { chmod -R a+rX \$\{VERA_DEV_ENV_BASE\}/gcc-5.4.0 }
$$

This should allow anyone to read (but not modify) any of the installed files and directories.

If the owning user is not vera-admin, then change the owning user to avoid accidental modifications by the original installer with the following command:

$$
\text { chown -R vera-admin <some-dir> }
$$

For example, if the original installer is not the current owning user, then the following commands can be run to transfer ownership to the vera-admin user:

chown $-\mathrm{R}$ vera-admin \$ $\{$ VERA_DEV_ENV_BASE $\} /$ gcc-5.4.0

This will avoid problems with accidental modifications to the installed directories by the original installer.

As an optional final step, to clean up disk space, users can delete the scratch space and the TPLs source repository by entering

$$
\begin{aligned}
& \mathrm{rm}-\mathrm{rf} \text { \$\{VERA_SCRATCH_DIR } \\
& \mathrm{rm}-\mathrm{rf} \text { \$\{VERA_BASE_DIR }\} / \text { vera_tpls }
\end{aligned}
$$

WARNING: Users should only remove these directories if they are sure that the VERA Dev Env is correctly installed and the necessary dependent VERA components are building and running correctly, at least related to the installed VERA Dev Env.

Only the local VERA source and build tree should remain locally:

$$
\text { \$\{VERA_BASE_DIR }\} /
$$

This can be used to clone and build VERA components with the installed VERA Dev Env. However, if VERA will no longer be built under this directory, then it can be removed with:

$$
\text { rm -rf \$ \{VERA_BASE_DIR\} }
$$

This would only leave the install of the VERA Dev Env under \$ VVERA_DEV_ENV_BASE\}/.

At this point, the VERA Dev Env would be considered successfully installed, so users of the dev env must source the script and update path for Anaconda2-for example:

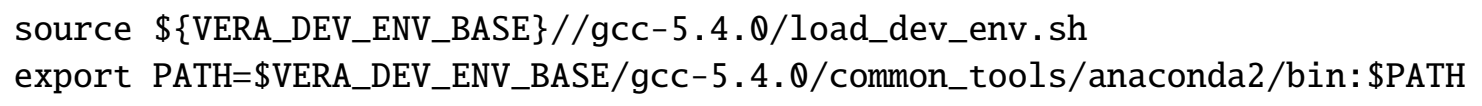

This is done in the .bash_profile file, for instance. 


\section{DETAILS ON INSTALLING VERA}

Once the VERA Dev Env is installed on a system and loaded into the user's shell environment, anyone with access to the VERA sources (either through a tarball or through the cloned Git repositories) can configure, build, test, and install the VERA components.

Information on the installation directory layout is provided at

\$\{VERA_DIR\}/doc/install/README.VERA

\subsection{GET SOURCE FOR VERA COMPONENTS TO INSTALL}

The process for obtaining the sources for the VERA components to install is identical to that for obtaining the sources to install and test the VERA Dev Env. This can be done simply with:

cd \$ $\{$ VERA_BASE_DIR $\}$ /

git clone git@code-int:VERA/VERA

cd VERA/

./clone_vera_repos.py

\subsection{CONFIGURE, BUILD, AND TEST VERA COMPONENTS TO INSTALL}

A configuration setting must be selected so that end users can build, test, and install various VERA components. For most systems, shared libraries are preferred because they typically use less disk space.

When installing VERA, it is important to build in a very shallow build directory-for example, using local scratch space to set up a build directory:

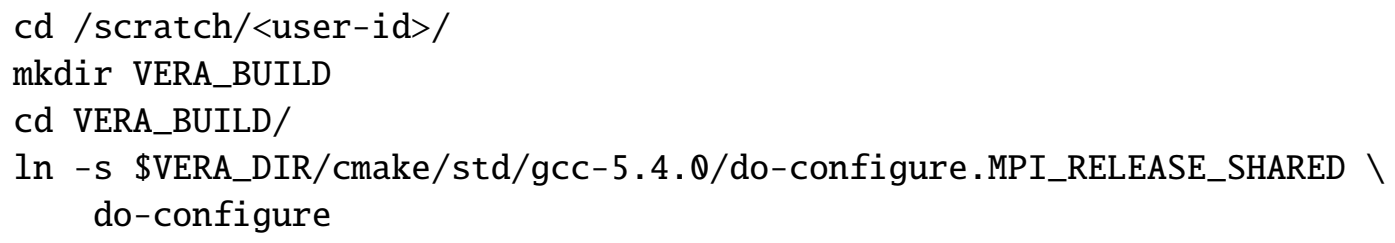

If a shallow build directory is not used, then CMake may fail to replace the RPATHs in the executables because the strings could be too long.

Once a do-configure script is set up, users must configure the system to point to the final install location. Assuming the target is

export VERA_INSTALL_DIR=/tools/vera_installs/`date +\%Y-\%m-\%d’

configure the install with

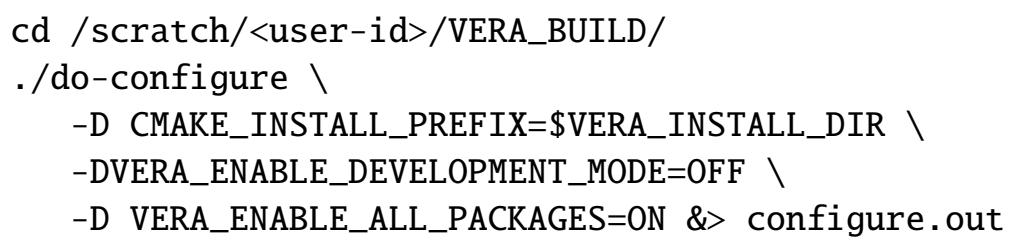


If the configure passed, then build and test using

make $-j 8 \&>$ make. out

ctest $-j 8 \&>$ ctest.out

(see Section 3.7).

All of the tests should pass, but if they do not, please email vera-support@ornl.gov and provide the tail of ctest. out and a copy of your do-configure script.

\subsection{INSTALL BUILT VERA COMPONENTS}

Before running make install, users may need to set up the base directory for the install by entering

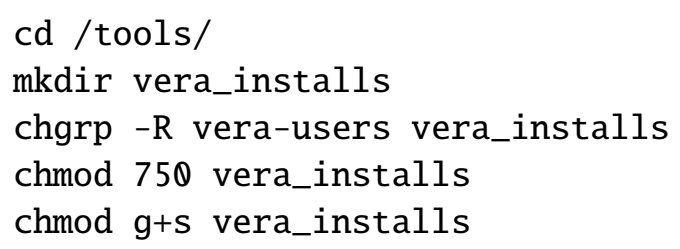

to protect VERA appropriately. (See the vera-users group described in Section 4.5.)

After a successful configure, build, and test has been performed, an install can be performed by entering

cd /scratch/<user-id>/VERA_BUILD/

umask 0007

make $-j 8$ install \&> make.install.out

Setting umask 0007 will ensure that only the vera-users group (or whatever it is called on the given system) has access to the installed VERA software.

This should set up an installation directory that looks like

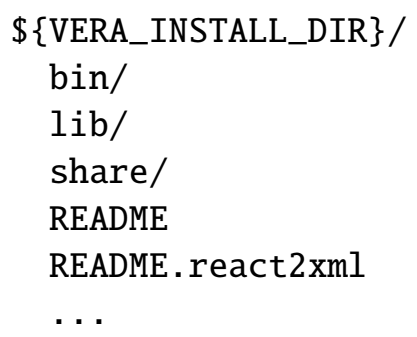

\subsection{DOCUMENTATION FOR INSTALLED VERA COMPONENTS}

Once VERA is installed, information on how to access the installed VERA components, along with their documentation and examples, can be found in the readme file:

\$\{VERA_INSTALL_DIR $/$

README 

APPENDIX A. ADDITIONAL INSTALLATION INFORMATION 



\section{A ADDITIONAL INSTALLATION INFORMATION}

\section{A.1 SET UP REMOTE SSH TUNNEL}

To access the Git repositories on code-int . ornl . gov when outside the ORNL network, an SSH tunnel must be set up through login1.ornl.gov. This requires the user to have an active UCAMS account with the 3-char $<$ ucams-id $>$. Once established, this SSH tunnel will set up a machine name called casl-dev on the local machine that can then be used in Git commands.

In the home directory, create the following file:

$$
\sim / \text {.ssh/config }
$$

This file contains

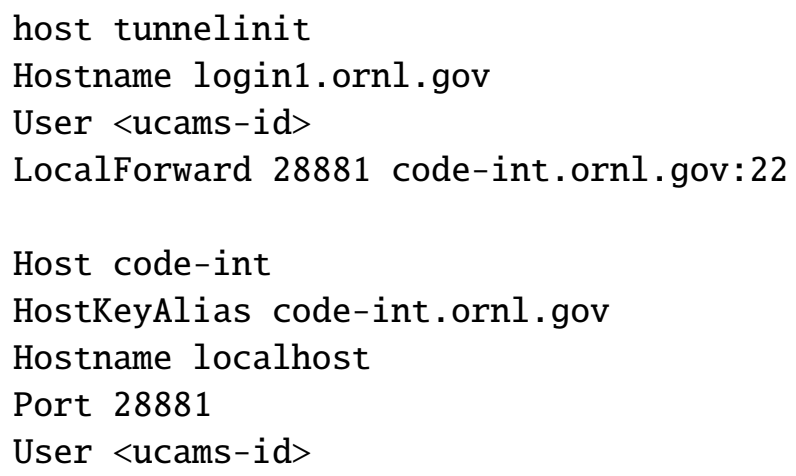

Please make sure to change the above port numbers to avoid conflict with other ports being used on the system or other SSH tunnels. If multiple users use the same port numbers, then collisions will occur, or the host machine will disallow the connection altogether.

To set up the SSH tunnel, in any terminal, type the command

$$
\text { ssh - fN tunnelinit }
$$

Each user will be prompted for a PASSCODE; this is the user's pin+6digits from their SecurID token.

The SSH tunnel will remain open for some amount of time and will continue to remain open if it is being actively used. However, it may be important in some cases to ensure that the tunnel is closed before logging off or performing other tasks. If a user creates an SSH tunnel, then the user should be able to close the SSH tunnel. Because the SSH tunnel is in the background, the following command should be used to find the SSH tunnel process:

ps aux | egrep ssh - fN tunnelinit

This will return the following type of response:

<ucams-id> $10535 \quad 0.3 \quad 0.0 \quad 66032 \quad 3804 ?$ ? Ss $11: 08 \quad 0: 19$ ssh -fN tunnelinit

The kill command can then be used to end the tunnel process:

kill $-9<$ process number $>$ 
In this case 10535 , the process number will always be the second item in the returned fields from the ps aux command.

This will close the SSH tunnel.

\section{A.2 MINIMAL SYSTEM PACKAGE SETUP ON VARIOUS SYSTEMS}

Package managers on different systems have different names for the needed packages they install, as described in Section 4.3.

The following table lists the names of the packages that must be installed on three different machines where VERA has been installed in the past. In addition, the last column specifies the executable that should be in the system's path if that the package is installed. Testing for the existence of these executables can be accomplished with the which command. For example:

which gcc

may return

/usr/bin/gcc

If a path to the executable is not returned by the which command, then the package is not installed.

\begin{tabular}{|l|l|l|l|l|}
\hline Package & CentOS 6.6/Redhat 6.4 & Ubuntu 14.04.1 & openSUSE 11.4 & Executables \\
\hline GCC C Compiler & gcc & gcc & gcc & gcc \\
\hline GCC C++ Compiler & gcc-c++ & g++ & g++ & gcc-c++ \\
\hline GNU M4 & m4 & M4 & M4 & m4 \\
\hline GNU texinfo & texinfo & texinfo & & makeinfo \\
\hline Git & git & git & git & git \\
\hline Python & python-dev & python-dev & python-dev & python-dev \\
\hline NumPy & numpy & python-numpy & python-numpy & $? ? ?$ \\
\hline Bash & bash & bash & bash & bash \\
\hline Perl & perl & perl-base & perl & perl \\
\hline X11 (dev) & libX11-devel & libx11-dev & & \\
\hline Xorg (dev) & xorg-x11-server-devel & xorg-dev & & \\
\hline Zlib (dev) & zlib-devel & zlib-dev & & \\
\hline libcurl (dev) & libcurl & curl & curl & \\
\hline
\end{tabular}

There are no executables for the X11, Xorg, or Zlib development libraries, and no executables are needed for the libcurl library. To verify that these packages are installed, users can search for a required library (in the user's LD_LIBRARY_PATH) or a required include file or directory in /usr/include. Suggested files/folders to check to verify installation of the X11, Xorg, and Zlib development libraries are provided in the following table.

On CentOS and Redhat systems, the command yum install <package > installs a package-for example:

sudo yum install gcc-c++ 


\begin{tabular}{|l|l|l|}
\hline Package & usr/lib or /usr/lib64 & /usr/include \\
\hline X11 (dev) & libX11.so & X11 \\
\hline Xorg (dev) & & xorg \\
\hline Zlib (dev) & libz.so & zlib.h \\
\hline Zlib (dev) & libcurl.so & curl/curl.h \\
\hline
\end{tabular}

On Ubuntu systems, the command apt-get install <package > installs a package—for example:

sudo apt-get install g++

On SUSE and openSUSE systems, the command zypper install <package> installs a package. For example:

sudo zypper install gcc-c++

A user with sudo privileges must install these packages because they are installed into the base system directories. However, it is possible to use some package managers to install missing packages to a different location that does not require root or sudo access. However, this is beyond the scope of this document. Consult the documentation for the package manager on your system for more information.

\section{A.3 OFFICIAL VERA TPL VERSIONS}

The source code for the official TPL versions that are installed using the install_tpls.sh tool (Section 3.6) are stored in the vera_tpls Git repository, and the current official versions are shown in the following table.

\begin{tabular}{|l|l|}
\hline Third Partly Library (TPL) & Version \\
\hline LAPACK & 3.3 .1 \\
\hline Boost & 1.55 .0 \\
\hline ZLib & 1.2 .7 \\
\hline HDF5 & 1.10 .2 \\
\hline NETCDF & 4.4 .1 \\
\hline PETSc & 3.6 .4 \\
\hline SLEPC & 3.6 .3 \\
\hline Silo & 4.10 .2 \\
\hline SUNDIALS & 2.9 .0 \\
\hline QT & 4.8 .7 \\
\hline
\end{tabular}

The exact version of TPLs matching this particular version of VERA is always indicated by the Git tag for the vera_tpls repository, as given in Section 3.6. 


\section{A.4 SHARED VS. STATIC LIBRARIES}

The default install of the VERA TPLs in Section 3.6 and VERA itself in Section 3.7 use shared libraries. However, these instructions also describe how to install static libraries. In general, users should prefer shared libraries over static libraries on most platforms: shared libraries use less disk space, link faster, and allow for quick bug fixes and simpler upgrades. However, if RPATH is not written into the executables and shared libraries, then users will have to set LD_LIBRARY_PATH pointing to the location of the correct shared libraries.

Moreover, some systems (especially some HPC machines) either require or strongly recommend the usage of static libraries. In addition, static libraries are more self-contained and are less sensitive to the machine environment from which they run and they never have RPATH issues. But executables built using static libraries take up far more disk space and take longer to link. 




(n)

